\title{
Toxicity and disease-related outcomes after radiotherapy for head and neck cancer in human immunodeficiency virus-positive patients
}

\author{
David J. Grew ${ }^{1}$, Benjamin T. Cooper ${ }^{1}$, Susanna Nguy $^{1}$, Jason Halperin ${ }^{2}$ and Nicholas J. Sanfilippo ${ }^{1}{ }^{*}$ \\ ${ }^{1}$ Department of Radiation Oncology, New York University School of Medicine, New York, NY, USA \\ ${ }^{2}$ Department of Infectious Disease, New York University School of Medicine, New York, NY, USA
}

Edited by:

Jesper Grau Eriksen, Odense

University Hospital, Denmark

\section{Reviewed by:}

Barbara Alicja Jereczek-Fossa, University of Milan, Italy Jesper Grau Eriksen, Odense

University Hospital, Denmark

\section{*Correspondence:}

Nicholas J. Sanfilippo, Department of Radiation Oncology, New York University School of Medicine, 160

East 34th Street, New York, NY 10016, USA

e-mail:nicholas.sanfilippo@

nyumc.org
Background: Human immunodeficiency virus (HIV) seropositivity may be associated with higher risk of local recurrence and poor survival in multiple malignancies. However, longterm disease control in HIV-positive patients with head and neck cancer (HNC) is not well described. The purpose of this study is to review the disease-related outcomes of HIVpositive patients who underwent radiotherapy (RT) or chemoradiotherapy (CRT) at our institution.

Methods: We retrospectively reviewed 24 HIV-positive patients who underwent RT for HNC between 2004 and 2013. Patient characteristics, treatment details, and outcomes were collected. Overall survival (OS) and local recurrence-free survival (LRFS) were investigated. Kaplan-Meier estimated survival was calculated.

Results: Median follow-up was 21 months. All patients were treated with curative intent. Eighty-three percent had stage III-IV. Primary sites of disease included oropharynx $(n=12)$, larynx $(n=6)$, oral cavity $(n=2)$, unknown primary $(n=2)$, nasal cavity $(n=1)$, and paranasal sinuses $(n=1)$. Four patients $(17 \%)$ had definitive RT alone and nine had definitive CRT (38\%; eight cisplatin and one cetuximab). Eleven (46\%) were treated in the adjuvant setting after surgical resection; six with RT alone and five with concurrent cisplatin. Eight patients had acute Grade 3 toxicity with no acute Grade 4 or 5 toxicities. Fifteen patients (63\%) were alive and disease-free. Two- and 5-year OS was 67 and 59\%, respectively. LRFS at 2 -years was $82 \%$. Median OS was 83 months.

Conclusion: In this cohort, HIV-positive patients treated aggressively with curative intent had excellent OS and local control following RT or CRT for HNC compared to historical controls. Treatment was relatively well tolerated. This group of patients should be managed aggressively with intent to cure.

Keywords: human immunodeficiency virus, head and neck cancer, radiotherapy, squamous cell carcinoma, oropharynx

\section{INTRODUCTION}

In the highly active anti-retroviral therapy (HAART) era, human immunodeficiency virus (HIV)-positive patients are living longer and are at higher risk for developing non-AIDS-defining malignancies (1-3). The incidence of head and neck cancer (HNC) in particular, has increased markedly since the widespread use of HAART (3-6). Despite becoming a more common problem, the optimal management of HNC in HIV-positive patients is unknown because of a paucity of data.

In HIV-positive patients with anal squamous cell carcinoma (SCC), some have argued against aggressive therapy in patients with advanced disease, citing concerns regarding increased toxicity after chemoradiation (CRT) $(7,8)$. The combined modality treatment of HNC with concurrent CRT in both the definitive and adjuvant setting results in improved disease outcomes at the cost of added toxicity (9-12). However, the magnitude of increased toxicity in HIV-positive patients is not well described. Some reports suggest that the acute toxicity of radiotherapy (RT) or CRT in HIV-positive HNC patients is comparable to that of HIV-negative patients (13-15). However, data from Mourad et al. suggest that RT is less tolerated among HIV-positive patients (16), possibly narrowing the therapeutic window in this fragile patient population.

Data on disease-related outcomes of HIV-positive patients with $\mathrm{HNC}$ are lacking. However, this information is essential for oncologists to effectively weigh the certainty of treatment toxicity against the potential benefit of aggressive treatment when making management decisions in this patient population. Furthermore, a better understanding of disease control and survival rates aids in counseling patients on reasonable treatment expectations. Here, we review our single institution experience treating HNC in HIV-positive patients over a 10 -year period and describe clinical 
outcomes. Secondary objectives include reporting acute toxicity, patterns of failure, and other factors, which may affect prognosis in $\mathrm{HIV}$-positive HNC patients.

\section{MATERIALS AND METHODS PATIENTS}

Approval of the Institutional Review Board was obtained for this retrospective study. All patients who received radiation therapy to the head and neck in our department between 2004 and 2014 were reviewed. Our radiation oncology department services patients from both a hospital that mainly accepts patients with private insurance and a public, community-based hospital, which is obligated to treat prisoners, underinsured, and uninsured patients. Patients from both institutions were included in this analysis. HIV-positive patients with cancer of the aerodigestive tract were identified. Two of 13 patients from our previous report on acute toxicity were excluded (squamous cell cancer of the skin and parotid hyperplasia) from this analysis (13). The remaining 11 patients and 13 additional patients were included and evaluated for long-term disease-related outcomes. Patient and treatmentrelated data that were examined included age, gender, histologic diagnosis, primary disease site, stage of disease, human papillomavirus (HPV) status, HAART therapy, RT technique [threedimensional conformal radiotherapy (3D-CRT) or intensity modulated radiotherapy (IMRT)], total RT dose, duration of RT, and the presence of concurrent chemotherapy. HIV-related information examined included pre-treatment CD4+ lymphocyte count and viral load. Patient toxicity data were manually garnered from the radiation and medical oncology treatment charts. All initial consultation, weekly radiation on-treatment, medical oncology on-treatment, and follow-up notes were examined. The electronic medical record of the referring hospital system was systematically queried in addition to the paper charts for every patient.

\section{TREATMENT AND FOLLOW-UP}

All treatments were delivered with megavoltage photon or electron beams. In patients receiving concurrent chemotherapy, cisplatin $100 \mathrm{mg} / \mathrm{m}^{2}$ was given every 3 weeks in all but one patient, who received cetuximab $400 \mathrm{mg} / \mathrm{m}^{2}$ loading dose followed by $250 \mathrm{mg} / \mathrm{m}^{2}$ weekly for the duration of radiation therapy. Patients were evaluated by a radiation oncologist on a weekly basis during RT, then monthly in multidisciplinary clinic with an otolaryngologist, radiation oncologist, and medical oncologist following RT completion for 3 months, then every 3 months. Acute and late toxicity was assessed as well as any treatment-related complications. Treatment outcomes that were examined included presence of local recurrence, distant metastasis, and death. HIV specific follow-up was at the discretion of the treating infectious disease specialist.

\section{STATISTICS}

Chi-square test was used to compare frequencies and $t$-test was used to compare means. Overall survival (OS) was defined as the duration from the start of RT to most recent follow-up or death. Local recurrence-free survival (LRFS) was defined as time from start of RT to most recent follow-up, tumor recurrence at the primary site or death. Survival estimates were calculated according to the Kaplan-Meier method. All statistical analyses were performed using SPSS version 20 (SPSS Inc., Chicago, IL, USA).

\section{RESULTS}

\section{PATIENT CHARACTERISTICS}

Twenty-four HIV-positive patients underwent RT for HNC of the aerodigestive tract. Median age was 53 (range, 32-67); 4 patients were women, 20 were men. Twenty-three patients had SCC, one patient had sarcomatoid SCC (Table 1). Primary disease sites included oropharynx $(n=12)$, larynx $(n=6)$, oral cavity $(n=2)$, unknown primary $(n=2)$, nasal cavity $(n=1)$, and paranasal sinuses $(n=1)$. Three patients $(13 \%)$ had stage II disease, $5(21 \%)$ had stage III, $14(58 \%)$ had IVA, and $2(8 \%)$ had IVB. Sixteen patients reported smoking ever; four patients reported $\geq 10$ pack-years smoking history.

\section{SURVIVAL}

At a median follow-up of 21 months, 16 patients $(67 \%)$ were alive and $15(63 \%)$ were alive and free of disease. Projected 2

\section{Table 1 | Patient and disease characteristics}

\begin{tabular}{|c|c|}
\hline Characteristic & Total patients $(\%)$ \\
\hline \multicolumn{2}{|l|}{ Gender } \\
\hline Male & $20(83)$ \\
\hline Female & $4(17)$ \\
\hline \multicolumn{2}{|l|}{ Histology } \\
\hline SCC & $23(96)$ \\
\hline Sarcomatoid SCC & $1(4)$ \\
\hline \multicolumn{2}{|l|}{ Primary site } \\
\hline Oropharynx & $12(50)$ \\
\hline Larynx & $6(25)$ \\
\hline Oral cavity & $2(8)$ \\
\hline Unknown primary & $2(8)$ \\
\hline Nasal cavity & $1(4)$ \\
\hline Paranasal sinuses & $1(4)$ \\
\hline \multicolumn{2}{|l|}{ T Stage } \\
\hline Tx & $1(4)$ \\
\hline TO & $2(8)$ \\
\hline $\mathrm{T} 1$ & $3(13)$ \\
\hline $\mathrm{T} 2$ & $7(29)$ \\
\hline T3 & $5(21)$ \\
\hline $\mathrm{T} 4$ & $6(25)$ \\
\hline \multicolumn{2}{|l|}{ N Stage } \\
\hline No & $8(33)$ \\
\hline N1 & $4(17)$ \\
\hline N2 & $10(42)$ \\
\hline N3 & $2(8)$ \\
\hline \multicolumn{2}{|c|}{ AJCC seventh edition stage group } \\
\hline II & $3(13)$ \\
\hline III & $5(21)$ \\
\hline IVA & $14(58)$ \\
\hline IVB & $2(8)$ \\
\hline
\end{tabular}

SCC, squamous cell carcinoma; AJCC, American Joint Committee on Cancer. 


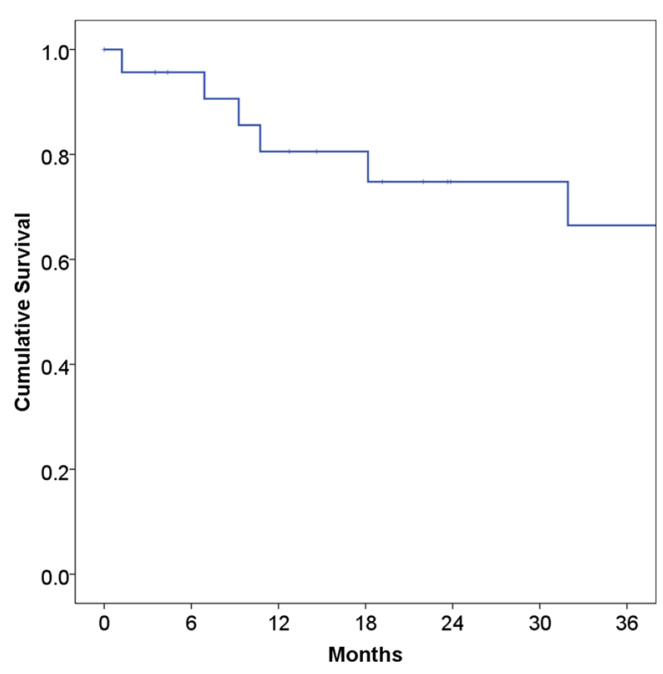

FIGURE 1 | Overall survival among human immunodeficiency virus-positive patients undergoing radiotherapy for head and neck cancer.

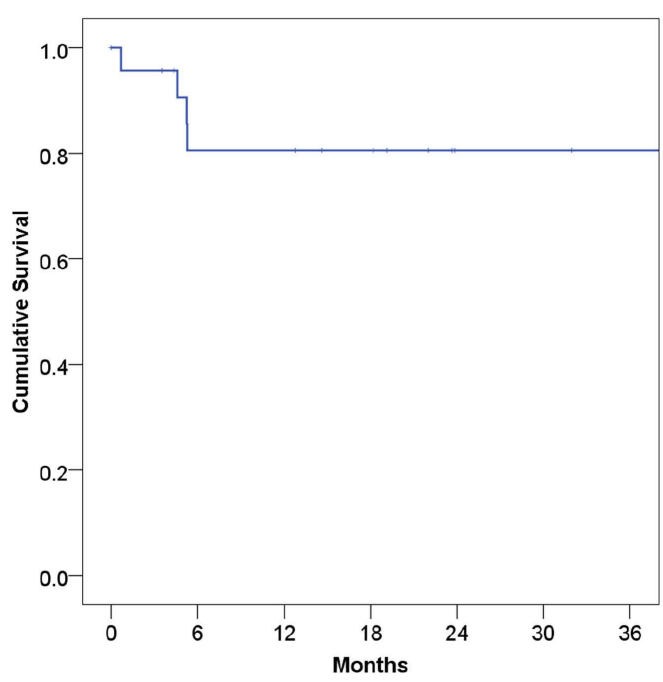

FIGURE 2 | Local recurrence-free survival among human immunodeficiency virus-positive patients undergoing radiotherapy for head and neck cancer.

and 5-year OS was 67 and 59\%, respectively (Figure 1). Median OS was 83 months (95\% CI 9.4-156 months). Causes of death include widespread metastases $(n=4)$, progression of primary $(n=2)$, sepsis during chemotherapy for second primary malignancy (lung) $(n=1)$, and unknown $(n=1)$. Of note, no patients died of complications from acquired immunodeficiency. Projected 2-year LRFS was 82\% (Figure 2), median LRFS was not reached. Of the patients who experienced local failure, primary sites of disease included base of tongue $(n=3)$ and retromolar trigone $(n=1)$.

\section{HIV-RELATED PARAMETERS}

Data on HAART were available for 22 patients, 19 (86\%) of which were on HAART at the time of consultation. All 19 patients on HAART continued therapy concurrently with RT. Twelve patients were taking protease inhibitors concurrently with RT. CD4+ lymphocyte counts were available for 12 patients. Median CD4+ cell count was 261 cells $/ \mu \mathrm{L}$ (range $71-858$ cells $/ \mu \mathrm{L}$ ). HIV viral load was available for 12 patients. Ten patients had an undetectable viral load ( $<50$ copies $/ \mathrm{mL}$ ), one had a viral load 687 copies $/ \mathrm{mL}$, and one patient not on HAART had viral load 46,600 copies/mL. HPV status was available for three patients, two of whom were HPVpositive. There was no significant difference in rates of death, local recurrence, or distance metastases among patients taking HAART medications. There was also no significant difference in the rate of death, local recurrence, or metastases among patients taking protease inhibitors.

\section{RADIOTHERAPY DETAILS}

Radiotherapy was delivered by 3D-CRT for 15 patients (63\%) and IMRT for 9 (37\%). Four patients (17\%) had definitive RT alone and nine CRT (38\%; eight cisplatin and one cetuximab). Eleven $(46 \%)$ were treated in the adjuvant setting after surgical resection, six with RT alone and five with concurrent cisplatin. Median RT dose for patients undergoing definitive treatment was $70 \mathrm{~Gy}$ (range 35-70.4 Gy) and median RT dose for patients undergoing postoperative treatment was 66 Gy (range 54-70 Gy). Three patients (13\%) terminated treatment prematurely. One patient had progression of disease and treatment was terminated at $35 \mathrm{~Gy}$. The patient expired 12 days later. Two patients stopped coming in for treatment mid-course and were lost to follow-up despite multiple phone calls and sending a certified letter. All patients were treated with conventional fractionation daily, 5 days a week. Two patients received $1.8 \mathrm{~Gy}$ per fraction, all others received $2 \mathrm{~Gy}$ per fraction. Median RT course duration was 53 days (range 21-71 days).

\section{HEMATOLOGIC DETAILS}

The median pre-treatment white blood cell count, absolute neutrophil count (ANC), hemoglobin level, and platelets were 6,100 cells $/ \mu \mathrm{L}, 3,700$ cells $/ \mu \mathrm{L}, 11.1 \mathrm{~g} / \mathrm{dL}$, and 240,000 cells $/ \mu \mathrm{L}$, respectively. The median nadir of the white blood cell count, ANC, hemoglobin level, and platelets were 2,200 cells $/ \mu \mathrm{L}, 1,200$ cells $/ \mu \mathrm{L}$, $8.9 \mathrm{~g} / \mathrm{dL}$, and 145,000 cells $/ \mu \mathrm{L}$, respectively. One patient receiving chemotherapy and one patient treated definitively with RT alone required hospitalization for neutropenic fever. The patient being treated with RT alone had local disease progression and eventually died of sepsis related to the progressive disease. This corresponded to an $8 \%$ rate of admission for neutropenic fever in the entire cohort. Eight of 24 patients became neutropenic $(\mathrm{ANC}<1,000$ cells $/ \mu \mathrm{L})$ at some point during their treatment, most of whom were treated with granulocyte colony-stimulating factor support. Interestingly, three of the patients who became neutropenic were not receiving chemotherapy.

\section{TOXICITY}

Grade 0,1 , and 2 toxicities are displayed in Table 2. There were no Grade 4 or 5 acute toxicities in this series. Eight patients (33\%) experienced Grade 3 toxicity: three had Grade 3 dermatitis, four 
Table 2 | Acute toxicity: RTOG common toxicity criteria and total numbers of patients affected.

\begin{tabular}{lccccc}
\hline Acute toxicity & Grade 0 & Grade 1 & Grade 2 & Grade 3 & Grade 4 \\
\hline Dermatitis & 1 & 7 & 5 & 3 & 0 \\
Mucositis & 2 & 3 & 8 & 4 & 0 \\
Fatigue & 16 & 0 & 1 & 0 & 0 \\
Esophagitis & 9 & 3 & 4 & 1 & 0 \\
Nausea & 14 & 1 & 1 & 0 & 0 \\
Anorexia & 14 & 2 & 1 & 0 & 0 \\
Xerostomia & 3 & 7 & 5 & 0 & 0 \\
\hline
\end{tabular}

RTOG, radiation therapy oncology group.

had Grade 3 mucositis, and one had Grade 3 esophagitis (Table 2). One patient with $\mathrm{T} 4 \mathrm{aN} 2 \mathrm{~b}$ base of tongue cancer had laryngeal edema necessitating an extended treatment break, resulting in a treatment time of 71 days total. Three patients had oropharyngeal thrush during RT, which resolved with topical antifungal treatment. Median weight loss in the entire cohort was $5.9 \mathrm{~kg}$ (range $2.3-13.6 \mathrm{~kg}$ ). Median weight loss in the patients treated with or without concurrent chemotherapy were 7.5 and $5.5 \mathrm{~kg}$, respectively. This corresponded to a weight loss as a percentage of total body weight $11.2 \%$ (range -8 to $30 \%$ ) in patients receiving concurrent chemotherapy compared to $7.4 \%$ (range 3-19\%) in patients without combined therapy. The only patient that gained weight during treatment ( $-8 \%$ weight loss) was because of PEG tube placement after a difficult post-operative course.

There were no significant increases in the frequency of Grade 3 toxicity among patients taking HAART. Severe late RT complications were rare. One patient had wound dehiscence after adjuvant RT for T1N2b tonsillar cancer. He did not receive concurrent chemotherapy and was not on HAART. One patient developed a tracheoesophageal fistula after adjuvant CRT to for T3N2c supraglottic larynx cancer. One patient T1N2a supraglottic larynx cancer treated with definitive CRT to 70 Gy was PEG-dependent until his death in 2010.

\section{DISCUSSION}

In our series, HIV-positive patients treated with definitive or adjuvant RT or CRT for HNC had $67 \%$ OS and $82 \%$ local control at a median follow-up of 2 years, comparing favorably with historical data. In addition, treatment was well-tolerated with limited acute toxicity and rare late complications. Further, while highly curable, HNC in HIV-positive patients remains potentially lifethreatening. In our series, three-quarters of all patient deaths were a result of local or distant progression of disease. These findings provide further evidence to support a curative approach to HIV-positive patients with HNC.

Historically, advanced $\mathrm{HNC}$ has been a difficult disease to manage, with morbid treatment and poor long-term disease-related outcomes. The expected 2-year OS and local control after definitive CRT for stage III-IV HNC in immunocompetent patients range from 40 to $75 \%$ and 60 to $80 \%$, respectively $(11,17,18)$. Results after surgical resection and adjuvant CRT are similar, with approximately $65-75 \%$ survival and $80-85 \%$ local control at 2 -years (9, 10). Klein et al. published the first report on disease outcomes among HIV-positive patients (14). While their study is limited by small sample size $(n=12)$, they report excellent results with $78 \%$ OS and 92\% local control at 3-years. More recently, Mourad et al. reported $69 \%$ locoregional control and 55\% OS at 4 years among 73 HIV-positive patients treated with definitive RT (19).

There are several possible explanations for these favorable results. First, we treated all patients aggressively, with multimodality treatment where appropriate. Our treatment strategy is not modified based on HIV status. While each patient is approached individually, our general practice is CRT for definitive treatment of stage III/IV HNC and adjuvant CRT for high-risk pathologic features including positive margins and extracapsular extension. These strategies were employed in the majority of patients on this study.

The epidemiology of HNC is changing, and favorable outcomes in our series may be a reflection of this. In recent years, the incidence of HPV-unrelated HNC has declined while HPV-associated $\mathrm{HNC}$ is on the rise (20). HPV-positive HNC patients have a significantly better prognosis than HPV-negative patients (21) and high rates of HPV-positivity in our cohort may contribute to the favorable results we observed. Unfortunately, because most patients in this series were treated before HPV testing became institutional policy, we were only able to obtain HPV status from pathologic specimens of three patients. Also, to complicate matters, both referring hospital's pathology departments were submerged in Fall of 2012 due to a weather related catastrophe, making retrospective HPV analysis of possible stored specimen impossible. There are, however, other characteristics that suggest that a high-HPVpositivity rate in this cohort including younger age at diagnosis (median 53 years), high proportion of oropharyngeal primary site $(50 \%)$, and lack of heavy smoking history ( $17 \%$ patients had $>10$ pack-years).

It is possible that the addition of protease inhibitors concurrent with RT contributed to improved outcomes. Protease inhibitors block the Akt signaling pathway and result in synergistic tumor killing when given concurrently with RT (22). In our series, the administration of concurrent protease inhibitor with RT did not result in either worse toxicity or improved disease-related outcomes. However, this analysis is limited by small patient numbers, making it difficult to draw meaningful conclusions based on these data. The impact of protease inhibitors on clinical outcomes requires further exploration as it may have important implications for the widespread application of this therapy for tumors of many disease sites (23).

Acute toxicity in this cohort compares favorably with historical controls. RT alone typically results in $<25 \%$ Grade 3 or 4 mucositis in immunocompetent patients. The addition of concurrent chemotherapy increases the risk of Grade 3-4 mucositis to $32-84 \%$ (24). Here, we observed $33 \%$ had any Grade 3 acute toxicity and $17 \%$ had Grade 3 mucositis. We did not report rates of late toxicity because follow-up notes did not consistently classify toxicity by RTOG Grade. We determined that retrospectively converting narrative descriptions of late effects from notes to RTOG Grade was an extrapolation that introduced a high degree of uncertainty and bias to these data.

Looking forward, HIV-positive HNC patients may be excellent candidates for molecular targeted therapy. The ongoing RTOG 
1016 study randomizes p16-positive HNC patients with clinical stage T1-2, N2a-3, or T3-4, any $\mathrm{N}$ to concurrent accelerated RT with either cisplatin or cetuximab. This head to head comparison will determine whether cetuximab results in equivalent survival as compared to cisplatin. The results of this trial are highly anticipated as they may usher in a new era in which chemotherapy is replaced by molecular targeted agents in HPV-positive HNC. This is especially relevant for HIV-positive HNC patients for whom epidemiologic data suggest high rates of HPV-related disease.

The strengths of this study are that it is a single institution experience over 10 years entirely in the HAART era with a consistent curative treatment approach. All patients were treated by the same two radiation oncologists. Limitations include retrospective analysis, which subjects this study to the typical biases, including selection bias. Additionally, as a retrospective study, it relies on the accuracy of follow-up clinic notes and imaging study reports for tabulation of toxicity and the classification of clinical endpoints. However, given the relative rarity of HNC in HIV-positive patients, prospective studies may not be feasible. Additionally, while this represents one of the largest series describing disease-related outcomes in this setting, patient numbers are small.

Finally, despite including 24 patients treated over the course of 10 years, median follow-up is only 21 months. This discordance between duration of the study period and median follow-up is a limitation of the study. A major contributor to the lack of follow-up is a large proportion of patients being referred from a hospital that serves uninsured patients of likely lower socioeconomic backgrounds who are less likely to complete routine follow-up as scheduled. Furthermore, lower rates of retention in the medical care system for HIV-positive patients have been documented and are associated with a lack of medical insurance, lower socioeconomic status, and competing psychosocial needs (25). Loss to follow-up for HIV specialty care is directly associated with increased mortality including death from malignancies (26). Those retained in the care of an infectious disease specialist have frequent follow-up with many health-care practitioners to manage HAART therapy and their other medical co-morbidities. This can lead to the misperception that sub-specialty follow-up is not required, which has been recently shown for missed gynecologic referrals in the setting of abnormal pap tests (27). This study highlights the challenge of establishing reliable follow-up with medical oncology, radiation oncology, and otolaryngology in this patient population, for whom other social issues may interfere with and take precedence over cancer surveillance.

\section{CONCLUSION}

In this cohort of HIV-positive patients with HNC, RT and CRT were well-tolerated and resulted in survival and disease control rates that compare favorably with historical controls. This group of patients should be managed aggressively with intention to cure. Despite excellent OS, most patient deaths that did occur were a result of disease progression. Premature de-escalation of therapy in this population may result in excess disease-related deaths.

\section{REFERENCES}

1. Deeken JF, Tjen ALA, Rudek MA, Okuliar C, Young M, Little RF, et al. The rising challenge of non-AIDS-defining cancers in HIV-infected patients. Clin Infect Dis (2012) 55(9):1228-35. doi:10.1093/cid/cis613

2. Herida M, Mary-Krause M, Kaphan R, Cadranel J, Poizot-Martin I, Rabaud $\mathrm{C}$, et al. Incidence of non-AIDS-defining cancers before and during the highly active antiretroviral therapy era in a cohort of human immunodeficiency virusinfected patients. J Clin Oncol (2003) 21(18):3447-53. doi:10.1200/JCO.2003. 01.096

3. Engels EA, Pfeiffer RM, Goedert JJ, Virgo P, McNeel TS, Scoppa SM, et al. Trends in cancer risk among people with AIDS in the United States 1980-2002. AIDS (2006) 20(12):1645-54. doi:10.1097/01.aids.0000238411.75324.59

4. Clifford GM, Polesel J, Rickenbach M, Dal Maso L, Keiser O, Kofler A, et al. Cancer risk in the Swiss HIV Cohort Study: associations with immunodeficiency, smoking, and highly active antiretroviral therapy. J Natl Cancer Inst (2005) 97(6):425-32. doi:10.1093/jnci/dji072

5. Patel P, Hanson DL, Sullivan PS, Novak RM, Moorman AC, Tong TC, et al. Incidence of types of cancer among HIV-infected persons compared with the general population in the United States, 1992-2003. Ann Intern Med (2008) 148(10):728-36. doi:10.7326/0003-4819-148-10-200805200-00005

6. Chaturvedi AK, Madeleine MM, Biggar RJ, Engels EA. Risk of human papillomavirus-associated cancers among persons with AIDS. J Natl Cancer Inst (2009) 101(16):1120-30. doi:10.1093/jnci/djp205

7. Chadha M, Rosenblatt EA, Malamud S, Pisch J, Berson A. Squamous-cell carcinoma of the anus in HIV-positive patients. Dis Colon Rectum (1994) 37(9):861-5. doi:10.1007/BF02052589

8. Holland JM, Swift PS. Tolerance of patients with human immunodeficiency virus and anal carcinoma to treatment with combined chemotherapy and radiation therapy. Radiology (1994) 193(1):251-4. doi:10.1148/radiology.193. 1.8090901

9. Cooper JS, Pajak TF, Forastiere AA, Jacobs J, Campbell BH, Saxman SB, et al. Postoperative concurrent radiotherapy and chemotherapy for highrisk squamous-cell carcinoma of the head and neck. N Engl J Med (2004) 350(19):1937-44. doi:10.1056/NEJMoa032646

10. Bernier J, Domenge C, Ozsahin M, Matuszewska K, Lefebvre JL, Greiner RH, et al. Postoperative irradiation with or without concomitant chemotherapy for locally advanced head and neck cancer. N Engl J Med (2004) 350(19):1945-52. doi:10.1056/NEJMoa032641

11. Adelstein DJ, Li Y, Adams GL, Wagner H Jr, Kish JA, Ensley JF, et al. An intergroup phase III comparison of standard radiation therapy and two schedules of concurrent chemoradiotherapy in patients with unresectable squamous cell head and neck cancer. J Clin Oncol (2003) 21(1):92-8. doi:10.1200/JCO.2003. 01.008

12. Brizel DM, Albers ME, Fisher SR, Scher RL, Richtsmeier WJ, Hars V, et al. Hyperfractionated irradiation with or without concurrent chemotherapy for locally advanced head and neck cancer. N Engl J Med (1998) 338(25):1798-804. doi:10.1056/NEJM199806183382503

13. Sanfilippo NJ, Mitchell J, Grew D, DeLacure M. Toxicity of head-and-neck radiation therapy in human immunodeficiency virus-positive patients. Int J Radiat Oncol Biol Phys (2010) 77(5):1375-9. doi:10.1016/j.ijrobp.2009.06.087

14. Klein EA, Guiou M, Farwell DG, Luu Q, Lau DH, Stuart K, et al. Primary radiation therapy for head-and-neck cancer in the setting of human immunodeficiency virus. Int J Radiat Oncol Biol Phys (2011) 79(1):60-4. doi:10.1016/j. ijrobp.2009.10.042

15. Kao GD, Devine P, Mirza N. Oral cavity and oropharyngeal tumors in human immunodeficiency virus-positive patients: acute response to radiation therapy. Arch Otolaryngol Head Neck Surg (1999) 125(8):873-6. doi:10.1001/archotol. 125.8.873

16. Mourad WF, Hu KS, Ishihara D, Shourbaji RA, Lin W, Kumar M, et al. Tolerance and toxicity of primary radiation therapy in the management of seropositive HIV patients with squamous cell carcinoma of the head and neck. Laryngoscope (2013) 123(5):1178-83. doi:10.1002/lary.23874

17. Forastiere AA, Goepfert H, Maor M, Pajak TF, Weber R, Morrison W, et al. Concurrent chemotherapy and radiotherapy for organ preservation in advanced laryngeal cancer. N Engl J Med (2003) 349(22):2091-8. doi:10.1056/ NEJMoa031317

18. Pignon JP, le Maitre A, Maillard E, Bourhis J; MACH-NC Collaborative Group. Meta-analysis of chemotherapy in head and neck cancer (MACH-NC): an 
update on 93 randomised trials and 17,346 patients. Radiother Oncol (2009) 92(1):4-14. doi:10.1016/j.radonc.2009.04.014

19. Mourad WF, Hu KS, Shasha D, Concert C, Ishihara D, Lin W, et al. Long-term outcome of seropositive HIV patients with head and neck squamous cell carcinoma treated with radiation therapy and chemotherapy. Anticancer Res (2013) 33(12):5511-6.

20. Chaturvedi AK, Engels EA, Anderson WF, Gillison ML. Incidence trends for human papillomavirus-related and -unrelated oral squamous cell carcinomas in the United States. J Clin Oncol (2008) 26(4):612-9. doi:10.1200/JCO.2007.14. 1713

21. Ang KK, Harris J, Wheeler R, Weber R, Rosenthal DI, Nguyen-Tan PF, et al. Human papillomavirus and survival of patients with oropharyngeal cancer. $N$ Engl J Med (2010) 363(1):24-35. doi:10.1056/NEJMoa0912217

22. Gupta AK, Cerniglia GJ, Mick R, McKenna WG, Muschel RJ. HIV protease inhibitors block Akt signaling and radiosensitize tumor cells both in vitro and in vivo. Cancer Res (2005) 65(18):8256-65. doi:10.1158/0008-5472.CAN-051220

23. Bernstein WB, Dennis PA. Repositioning HIV protease inhibitors as cancer therapeutics. Curr Opin HIV AIDS (2008) 3(6):666-75. doi:10.1097/COH. 0b013e328313915d

24. Trotti A. Toxicity in head and neck cancer: a review of trends and issues. Int $J$ Radiat Oncol Biol Phys (2000) 47(1):1-12. doi:10.1016/S0360-3016(99)00558-1

25. Thompson MA, Mugavero MJ, Amico KR, Cargill VA, Chang LW, Gross R, et al. Guidelines for improving entry into and retention in care and antiretroviral adherence for persons with HIV: evidence-based recommendations from an international association of physicians in AIDS care panel. Ann Intern Med (2012) 156(11):817-33. doi:10.7326/0003-4819-156-11-201206050-00419
26. Mugavero MJ, Lin HY, Willig JH, Westfall AO, Ulett KB, Routman JS, et al. Missed visits and mortality among patients establishing initial outpatient HIV treatment. Clin Infect Dis (2009) 48(2):248-56. doi:10.1086/595705

27. Wigfall LT, Bynum SA, Brandt HM, Friedman DB, Bond SM, Lazenby GB, et al. Cervical cancer prevention knowledge and abnormal pap test experiences among women living with HIV/AIDS. J Cancer Educ (2014). doi:10.1007/ s13187-014-0688-9

Conflict of Interest Statement: The authors declare that the research was conducted in the absence of any commercial or financial relationships that could be construed as a potential conflict of interest.

Received: 30 September 2014; accepted: 24 October 2014; published online: 10 November 2014.

Citation: Grew DJ, Cooper BT, Nguy S, Halperin J and Sanfilippo NJ (2014) Toxicity and disease-related outcomes after radiotherapy for head and neck cancer in human immunodeficiency virus-positive patients. Front. Oncol. 4:316. doi: 10.3389/fonc.2014.00316

This article was submitted to Head and Neck Cancer, a section of the journal Frontiers in Oncology.

Copyright (C) 2014 Grew, Cooper, Nguy, Halperin and Sanfilippo. This is an openaccess article distributed under the terms of the Creative Commons Attribution License (CC BY). The use, distribution or reproduction in other forums is permitted, provided the original author(s) or licensor are credited and that the original publication in this journal is cited, in accordance with accepted academic practice. No use, distribution or reproduction is permitted which does not comply with these terms. 